\title{
Robustness of mathematical models for biological systems
}

\author{
Tianhai Tian* \\ (Received 8 August 2003; revised 24 January 2004)
}

\begin{abstract}
The robustness of mathematical models for biological systems is studied by sensitivity analysis and stochastic simulations. Using a neural network model with three genes as the test problem, we study robustness properties of synthesis and degradation processes. For single parameter robustness, sensitivity analysis techniques are applied for studying parameter variations and stochastic simulations are used for investigating the impact of external noise. Results of sensitivity analysis are consistent with those obtained by stochastic simulations. Stochastic models with external noise can be used for studying the robustness not only to external noise but also to parameter variations. For external noise we also use stochastic models to study the robustness of the function of each gene and that of the system.
\end{abstract}

*Advanced Computational Modelling Centre, Department of Mathematics, The University of Queensland, Brisbane, Queensland 4072, Australia.

mailto:tian@maths .uq. edu . au

See http://anziamj.austms.org.au/V45/CTAC2003/Tia2 for this article, (C) Austral. Mathematical Soc. 2004. Published June 30, 2004. ISSN 1446-8735 


\section{Contents}

1 Introduction

C566

2 Methods for studying robustness

C567

3 Neural network models

C569

4 Single parameter robustness

C570

5 Robustness to multi-parameters

C574

6 Conclusions

C576

References

C577

\section{Introduction}

The notion of robustness in biological systems has received considerable interest recently by experiments and theoretical study. By saying that a system is robust we imply that a particular function or characteristic of the system is preserved despite changes in the operating environment [6] or genetic changes in its components [5]. For example, by a computer model [2] and later by experiments [1], it has been demonstrated that the adaptation mechanism found in the chemotactic signaling pathway in Escherichia coli is robust. For robust biological systems, we expect that mathematical models that attempt to explain these systems should also be robust. It has been proposed that the robustness of mathematical models be used as a criterion for determining plausibility of candidate models $[7,9]$.

One topic in the robustness analysis of mathematical models is the model's sensitivity to parameter variations. These variations may be errors in parameter estimation or changes in the components of biological systems. This 
topic has been studied by the sensitivity analysis techniques $[3,6,7]$. There are two approaches for the sensitivity analysis of single parameter: repeated simulations by varying one parameter while holding all others fixed; and adjacent models for comparing the importance of each parameter variation. However, single parameter insensitivity may not be sufficient owing to interactions between several parameters. Systematic changes of many parameters at a time suffer from an exponential increase in the number of parameters that need to be changed. More sophisticated methods are needed for studying multi-parameter robustness.

Another important topic is the robustness analysis of mathematical models for the noise in biological systems. Noise existing in biological systems is classified as external noise due to environmental fluctuations or internal noise due to small numbers of some important regulatory molecules [4]. As living systems are optimized to function in the presence of stochastic fluctuations, mathematical models for biochemical networks must withstand considerable variations and random perturbations of biochemical parameters [8]. The study of the robustness to noise normally is carried out by simulations of stochastic models $[8,9]$.

We investigate the robustness of mathematical models to external noise and the relationship between the robustness to parameter variations and that to external noise. In Section 2 we present adjacent models and stochastic models for studying robustness properties. A neural network model with three genes is introduced in Section 3. In Section 4 we discuss single parameter robustness and the discussion for multi-parameters is presented in Section 5 .

\section{Methods for studying robustness}

In this section we present methods used in this paper for studying the robustness of mathematical models. The first method is the adjacent model 
for studying parameter variations. For a given model (the base model) with parameter $p$

$$
\frac{d x}{d t}=f(t, x, p),
$$

we consider the solution $x^{*}$ of this system with a perturbed parameter $p+\Delta p$. The difference between solutions $x^{*}$ and $x$ is

$$
\frac{d\left(x^{*}-x\right)}{d t}=f\left(t, x^{*}, p+\Delta p\right)-f(t, x, p) \approx \frac{\partial f}{\partial x}\left(x^{*}-x\right)+\frac{\partial f}{\partial p} \Delta p .
$$

Together with the base model (1), the adjacent model for parameter $p$ is

$$
\frac{d \mathrm{Ep}}{d t}=\frac{\partial f}{\partial x} \mathrm{Ep}+\frac{\partial f}{\partial p} .
$$

Here Ep represents the drift of the solution with a unit parameter perturbation. The solutions of adjacent models for certain important parameters in the base model give insight into which parameter induces the largest error in solutions and when errors will be the largest in simulations.

In order to study external noise, Hasty et al. [4] introduced stochastic models by adding additive or multiplicative noise to mathematical models. For example, for a biological system with mathematical model (the base model)

$$
\frac{d x}{d t}=f(t, x)-d_{x} x
$$

the stochastic model with additive noise is

$$
d x=f(t, x) d t-d_{x} x d t+k d W(t) .
$$

When considering noise in the degradation process, the stochastic model with multiplicative noise is

$$
d x=f(t, x) d t-d_{x} x d t+k d_{x} x d W(t) .
$$

Here $W(t)$ is the Wiener process whose increments are independent Gaussian random variables, and $k$ is a scalar for adjusting the magnitude of noise. 
Based on a large number of stochastic simulations, moments of the simulated solutions can be calculated. Then the robustness to noise can be measured by the comparison of these moments with the solution obtained by the base model and with the variance of noise in model (3) or (4).

\section{$3 \quad$ Neural network models}

Neural network models have been used for expressing regulatory mechanisms in genetic regulatory networks [10]. A system with $N$ genes is

$$
\frac{d x_{i}}{d t}=s_{i} g_{i}(t)-d_{i} x_{i}, \quad \text { with } i=1, \ldots, N .
$$

Here $x_{i}, s_{i}$ and $d_{i}$ are the expression level, synthesis rate and degradation rate of gene $i$ in the system, respectively. The functions $g_{i}$ are the sigmoidal transfer function

$$
g_{i}(t)=\frac{1}{1+\exp \left[-r_{i}(t)\right]},
$$

with

$$
r_{i}(t)=\sum_{j=1}^{N} w_{i j} x_{j}(t)+b_{i} .
$$

Here $\boldsymbol{b}=\left(b_{i}\right)_{N}$ is a vector for reaction delay, and the weight matrix $\boldsymbol{w}=$ $\left(w_{i j}\right)_{N \times N}$ defines regulatory interactions between genes. A regulation from gene $j$ to gene $i$ means a non-zero weight $w_{i j}$. A positive weight implies a stimulating effect (positive feedback) while a negative weight implies repression (negative feedback). A zero weight means no regulatory interaction.

Figure 1 gives a gene network with three genes which has been used for realizing two important expression patterns: oscillation and steady state [10]. In this network, the product of Gene $A$ controls the expression of Gene $B$, which initiates the expression of Gene $C$. Gene $B$ induces the expression of 


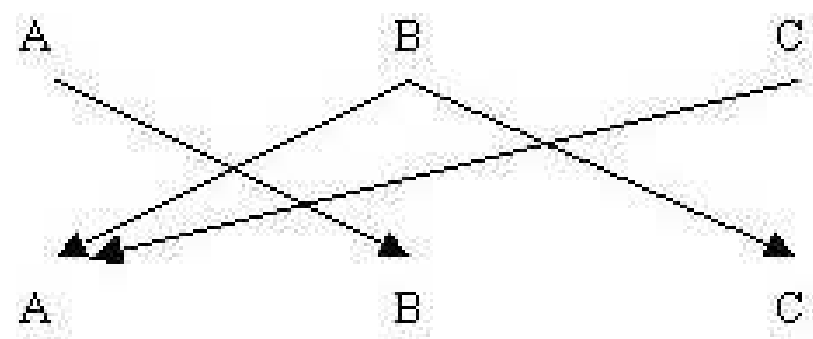

Figure 1: A gene network with three genes.

Gene $A$ forming a positive feedback. Gene $C$ in turn negatively controls the expression of Gene $A$, forming a negative feedback. The regulation in this network is characterized by the weight matrix [10]

$$
\boldsymbol{w}=\left[\begin{array}{ccc}
0 & 10 & -10 \\
10 & 0 & 0 \\
0 & 10 & 0
\end{array}\right]
$$

We realize a simulation with the steady state expression pattern by using (7) and

$$
\boldsymbol{s}=(1,1,1), \quad \boldsymbol{d}=\boldsymbol{s}, \quad \boldsymbol{b}=-\boldsymbol{s} ;
$$

and a simulation with the oscillatory expression pattern by using (7) and

$$
\boldsymbol{s}=(4.5,1,1), \quad \boldsymbol{d}=(0.6,1,1), \quad \boldsymbol{b}=-(3,3,3) .
$$

\section{Single parameter robustness}

In this section we will study the robustness of model (5) to parameter variations and fluctuations to each synthesis rate and degradation rate. For each synthesis rate $s_{i}(i=1,2,3)$, the adjacent model (together with (5)) is

$$
\frac{d \mathrm{Es}_{i j}}{d t}=s_{j} g_{j}^{2}(t) * e^{-r_{j}(t)} *\left[w(j,:) \cdot \mathbf{E s}_{i}\right]-d_{j} \mathrm{Es}_{i j}+\delta_{i j} g_{j}(t),
$$


with $j=1,2,3$. Here $\mathbf{E s}_{i}=\left(\operatorname{Es}_{i 1}, \operatorname{Es}_{i 2}, \operatorname{Es}_{i 3}\right)^{\top}$. For each degradation rate $d_{i}$ $(i=1,2,3)$, the adjacent model (together with (5)) is

$$
\frac{d \mathrm{Ed}_{i j}}{d t}=s_{j} g_{j}^{2}(t) * e^{-r_{j}(t)} *\left[w(j,:) \cdot \mathbf{E d}_{i}\right]-d_{j} \operatorname{Ed}_{i j}-\delta_{i j} d_{j},
$$

with $j=1,2,3$. Here $\operatorname{Ed}_{i}=\left(\operatorname{Ed}_{i 1}, \operatorname{Ed}_{i 2}, \operatorname{Ed}_{i 3}\right)^{\top}$.

Stochastic models for studying external noise in each reaction rate can be constructed by adding a stochastic process to the corresponding reaction rate. Similar to model (4), the stochastic model for studying fluctuations in each $s_{i}(i=1,2,3)$ takes the form

$$
d x_{j}=s_{j} g_{j}(t) d t+\delta_{i j} k s_{j} g_{j}(t) d W(t)-d_{j} x_{j} d t,
$$

with $j=1,2,3$. Here $k$ is a scalar for adjusting the magnitude of noise in the synthesis process. The stochastic model for each $d_{i}(i=1,2,3)$ is

$$
d x_{j}=s_{j} g_{j}(t) d t-d_{j} x_{j} d t-\delta_{i j} k d_{j} x_{j} d W(t),
$$

with $j=1,2,3$.

Figure 2 gives simulations of $\operatorname{Es}_{i 3}(i=1,2,3)$ from adjacent models (8) for the steady state expression pattern (left) and the oscillatory expression pattern (right). Variations in parameter $s_{3}$ have more influence on simulation results. Compared with the steady state pattern, parameter variations in the oscillatory pattern have much larger influence on network behaviour. Similar simulations can be obtained from adjacent models for degradation rates (9) but they are not presented here due to the limit of space. Similarly variations in parameter $d_{3}$ have more influence on simulation results and the oscillatory pattern is more sensitive to variations in degradation rates.

For stochastic model (10) with same $k=0.15$ for each $i(i=1,2,3)$, Figure 3 gives simulations of $x_{3}$ for the steady state expression pattern (left) and the oscillatory pattern (right). Similar to Figure 2, noise related to 

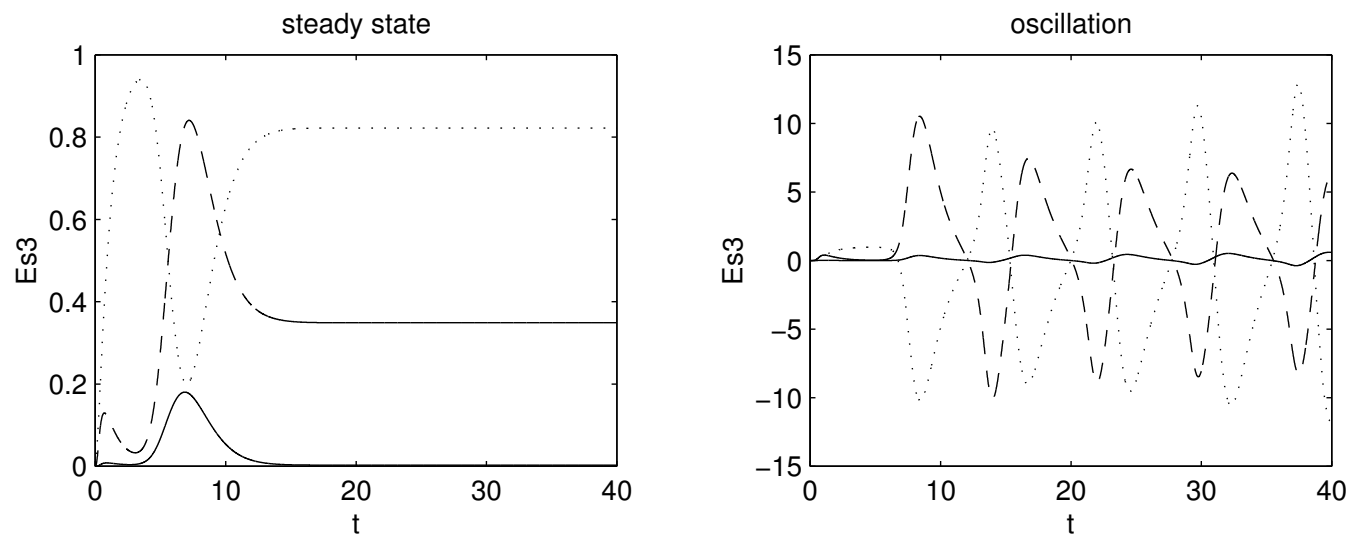

Figure 2: Simulations of $\mathrm{Es}_{i 3}$ for the steady state pattern (left) and the oscillatory pattern (right). (Es ${ }_{13}$ : line, $\mathrm{Es}_{23}$ : dash, $\mathrm{Es}_{33}$ : dot)
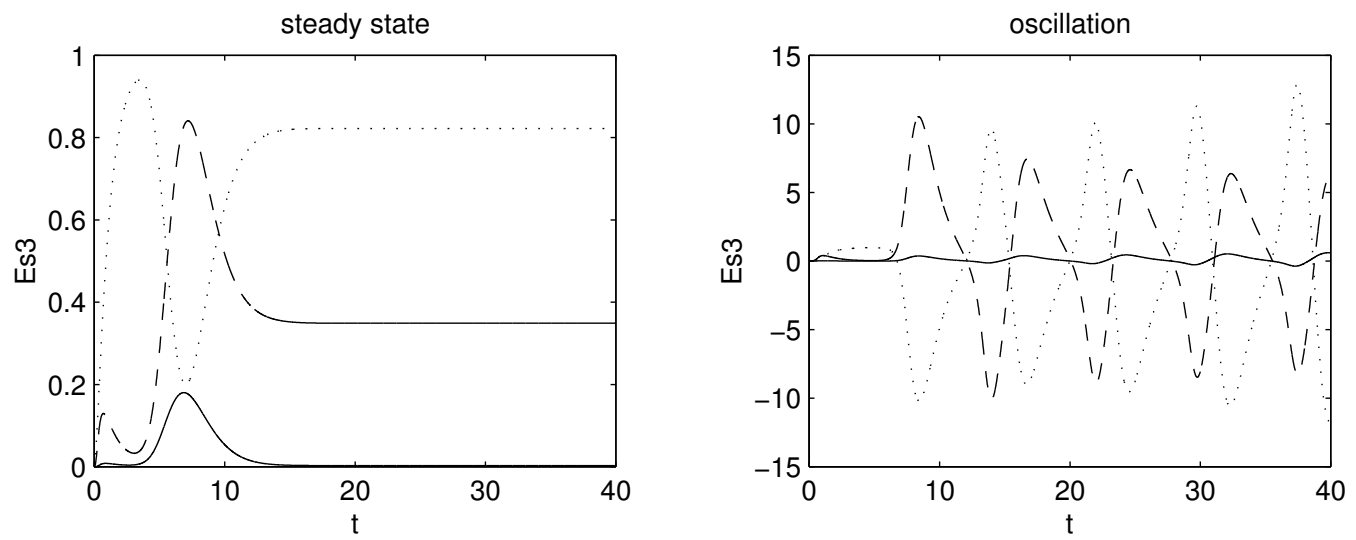

Figure 3: Simulations of $x_{3}$ from model (10) for the steady state pattern (left) and oscillatory pattern (right). (model with noise in $s_{1}$ : line, $s_{2}$ : dash, $s_{3}$ : dot). 
$s_{3}$ or $d_{3}$ has more influence on the system. In addition, fluctuations in the oscillatory pattern are much larger than those in the steady state pattern.

In addition to the qualitative results in Figures 2 and 3, we give quantitative results of the robustness properties of model (5). For the steady state expression pattern, we are interested in the drift of expression levels for the deterministic model

$$
D_{j}(p)=x_{j}(p)-x_{j},
$$

where $x_{j}$ is the expression level of gene $j$ in system $(5), x_{j}(p)$ is the expression level obtained from (5) in which parameter $p$ is perturbed. Based on $N$ stochastic simulations, we use the mean of drifts and standard deviation of $x_{j}$,

$$
\begin{aligned}
M D_{j}(p) & =\frac{1}{N} \sum_{k=1}^{N}\left[x_{j}^{(k)}(p)-x_{j}\right] \equiv \bar{x}_{j}(p)-x_{j}, \\
S D_{j}(p) & =\sqrt{\frac{1}{N-1} \sum_{k=1}^{N}\left(x_{j}^{(k)}(p)-\bar{x}_{j}(p)\right)^{2}},
\end{aligned}
$$

to measure the robustness properties to external noise. Here $x_{j}{ }^{(k)}(p)$ is the expression level of $x_{j}$ in the $k$ th simulation. It is obtained from a stochastic model with a stochastic component in parameter $p$.

Table 1 gives quantitative results from the deterministic and stochastic simulations for the steady state expression pattern. For the deterministic model (5), we vary each $s_{i}(i=1,2,3)$ by $s_{i}^{*}=s_{i}(1+0.15)$ and fix all other parameters. Similar considerations are applied to each $d_{i}$. The first row of Table 1 gives drifts of $x_{3}$ with each perturbed parameter. Results in the last two rows are based 1000 simulations from models (10) and (11). We use the same $k=0.15$ in noise terms of these stochastic models. These data give quantitative evidence to support the results in Figures 2 and 3.

The drifts of $x_{3}\left(D_{3}(p)\right)$ obtained by deterministic simulations are consistent with the means of drifts $\left(M D_{3}(p)\right)$ obtained by stochastic simulations. 
TABLE 1: Simulation results of $x_{3}$ for single parameter robustness

\begin{tabular}{|c|ccc|ccc|}
\hline & $p=s_{1}$ & $p=s_{2}$ & $p=s_{3}$ & $p=d_{1}$ & $p=d_{2}$ & $p=d_{3}$ \\
\hline$D_{3}(p)$ & $2.9 \mathrm{E}-4$ & $3.1 \mathrm{E}-2$ & $8.3 \mathrm{E}-2$ & $-2.6 \mathrm{E}-4$ & $-3.4 \mathrm{E}-2$ & $-7.4 \mathrm{E}-2$ \\
$M D_{3}(p)$ & $-9.1 \mathrm{E}-6$ & $-2.1 \mathrm{E}-3$ & $4.7 \mathrm{E}-3$ & $8.6 \mathrm{E}-6$ & $-2.1 \mathrm{E}-3$ & $7.4 \mathrm{E}-3$ \\
$S D_{3}(p)$ & $1.8 \mathrm{E}-4$ & $2.6 \mathrm{E}-2$ & $8.9 \mathrm{E}-2$ & $1.9 \mathrm{E}-4$ & $2.5 \mathrm{E}-2$ & $9.1 \mathrm{E}-2$ \\
\hline
\end{tabular}

This observation suggests that stochastic simulations can study robustness properties not only for external noise but also for parameter variations. In the following section we use stochastic simulations to measure the robustness properties of model (5) to multiple parameters.

\section{Robustness to multi-parameters}

For multi-parameter problems we do not address all of the possible combinations of the parameters. Instead we just study the robustness to noise in synthesis and degradation processes of each gene. The following models will be used in stochastic simulations:

$$
d x_{j}=\left[s_{j} g_{j}(t)-d_{j} x_{j}\right] d t+\delta_{i j}\left[k_{1} s_{j} g_{j}(t) d W_{1}-k_{2} d_{j} x_{j} d W_{2}\right],
$$

with $j=1,2,3$ for each gene $i(i=1,2,3)$. Here we use $k_{1}=k_{2}=0.15$.

Based on 1000 simulations, we calculate moments of the expression levels obtained from (12). The definitions for the mean of drifts and standard deviation of $x_{j}$ are defined by

$$
\begin{aligned}
M D_{j}(i) & =\frac{1}{N} \sum_{k=1}^{N}\left[x_{j}^{(k)}(i)-x_{j}\right] \equiv \bar{x}_{j}(i)-x_{j}, \\
S D_{j}(i) & =\sqrt{\frac{1}{N-1} \sum_{k=1}^{N}\left(x_{j}{ }^{(k)}(i)-\bar{x}_{j}(i)\right)^{2}}
\end{aligned}
$$


TABle 2: Simulation results of $x_{3}$ by (12) with noise in $s_{i}$ and $d_{i}$

\begin{tabular}{|c|ccc|}
\hline & $i=1$ & $i=2$ & $i=3$ \\
\hline$M D_{3}(i)$ & $4.64 \mathrm{E}-6$ & $5.9 \mathrm{E}-3$ & $7.1 \mathrm{E}-3$ \\
$S D_{3}(i)$ & $2.78 \mathrm{E}-4$ & $3.74 \mathrm{E}-2$ & 0.1324 \\
\hline
\end{tabular}

where $x_{j}{ }^{(k)}(i)$ is the expression level of $x_{j}$ in the $k$ th simulation. It is obtained from model (12) with stochastic components in $s_{i}$ and $d_{i}$. For the steady state expression pattern, Table 2 lists the means of drifts and standard deviations of $x_{3}$. Noise in $s_{1}$ and $d_{1}(i=1)$ has less impact on the system due to simple functions of Gene 1 (only stimulating Gene 2). For Gene $2(i=2)$, fluctuations of the system are larger than those of Gene 1 because of more functions of Gene 2 (stimulating both Gene 1 and Gene 3). The system with noise in $s_{3}$ and $d_{3}(i=3)$ is still robust but numerical simulations have the largest fluctuations. This is due to the critical negative feedback function of Gene 3 in the system. These results indicate that the influence of a gene on robustness properties depends on its functions in the system.

Finally, the robustness of model (5) to external noise which is related to the synthesis and degradation processes of all genes. This stochastic model has been studied in [9], with $j=1,2,3$

$$
d x_{j}=\left[s_{j} g_{j}(t)-d_{j} x_{j}\right] d t+k_{1 j} s_{j} g_{j}(t) d W_{1 j}-k_{2 j} d_{j} x_{j} d W_{2 j} .
$$

Figure 4 gives simulations of model (13) for the steady state pattern (left) and the oscillatory pattern (right) with $k_{1 j}=k_{2 j}=0.15(j=1,2,3)$. The steady state pattern is robust but the oscillatory pattern is strongly influenced by external noise. The period and amplitude of oscillations fluctuate widely in time. This result serves as an evidence for questioning the suitability of neural network models for describing oscillatory phenomena in genetic regulatory networks. 

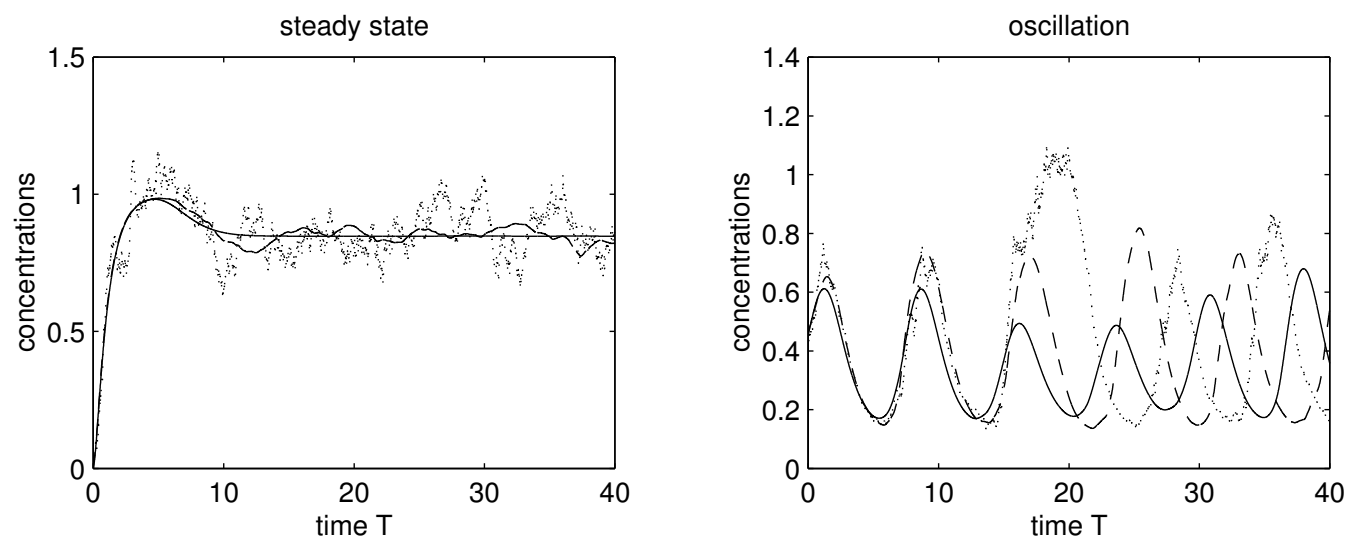

Figure 4: Simulations of model (13) for the steady state pattern (left) and the oscillatory pattern (right). ( $x_{1}$ : line, $x_{2}$ : dash, $x_{3}$ : dot).

\section{Conclusions}

For studying the robustness of mathematical models for biological systems, we should address three sources of uncertainty: errors in estimated parameters; external noise for environmental fluctuations; and internal noise due to small numbers of regulatory molecules. The analysis for each source of uncertainty is the first step in finding robustness properties of mathematical models. It would be very important to search relationships between the robustness properties of each source and the impact of combined sources on mathematical models. The work in this paper is an attempt in this direction. It is suggested that stochastic simulations be used to study the robustness not only to external noise but also to parameter variations. In this paper a neural network model with three genes has been used as the test problem. Future work will be based on the applications of stochastic simulations to more complicated biological systems. 


\section{References}

[1] U. Alon, M. G. Surette, N Barkai and S. Leibler. Robustness in bacterial chemotaxis. Nature, 397:168-171, 1999. C566

[2] N. Barkai, S. Leibler. Robustness in simple biochemical networks. Nature 387:913-917, 1997. C566

[3] R. S. Erb and G. S. Michaels. Sensitivity of biological models to errors in parameter estimates. Pacific Symposium on Biocomputing, 4:53-64, 1999. C567

[4] J. Hasty, J. Pradines, M. Dolnik and J. J. Collins. Noise-based switches and amplifiers for gene expression. Proc. Natl. Acad. Sci., 97:2075-2080, 2000. C567, C568

[5] J. W. Little, D. P. Shepley and D. W. Wert. Robustness of a gene regulatory circuit. EMBO J., 18:4299-4307, 1999. C566

[6] L. Ma and P. A. Iglesias. Quantifying robustness of biochemical network models. EMC Bioinformatics, 3:38, 2002. C566, C567

[7] M. Morohashi, et al. Robustness as a measure of plausibility in models of biochemical networks. J. Theor. Biol., 216: 19-30, 2002. C566, C567

[8] M. Thattai and A. van Oudenaarden. Intrinsic noise in gene regulatory networks. Proc. Natl. Acad. Sci., 98: 8614-8619, 2001. C567

[9] T. Tian and K. Burrage. Stochastic neural network models for genetic regulatory networks. Proceedings of the 2003 Congress of Evolutionary Computation 162-169, IEEE Press, 2003. C566, C567, C575

[10] J. Vohradsky. Neural network model of gene expression. FASEB J., 15:846-854, 2001. C569, C570 